\title{
A Hierarchical IRT Model for Criterion-Referenced Measurement
}

\author{
Rianne Janssen \\ Francis Tuerlinckx \\ Michel Meulders \\ Paul De Boeck \\ University of Leuven
}

Keywords: hierarchical IRT, criterion-referenced measurement, standard setting, Gibbs sampler, posterior predictive checks

A hierarchical IRT model is proposed for mastery classification in criterionreferenced measurement. In this model, items measuring the same criterion are grouped, and a difficulty and discrimination parameter of the criterion is estimated on the same scale as the person and item parameters. The level of proficiency of a student with respect to the criterion is determined by the probability of success on the criterion. Cutoff points on the probability scale can be used to classify respondents into masters and nonmasters. The hierarchical IRT model is estimated using the Gibbs sampler and tested using posterior predictive checks. The model is illustrated with a test measuring the attainment targets of reading comprehension (in Dutch) at the end of primary education.

An assumption often made in mastery learning is that mastering a trait or educational objective is a gradual process consisting of a progression along a continuum. A cutoff point on the continuum indicates the point of sufficient proficiency for a student to be classified as a master. This continuum view of

This paper was prepared under various grants to the respective authors. The first author worked as a postdoctoral assistant at the Leuven Institute for Educational Research and under a postdoctoral research grant from the University of Leuven (PDM/97/042). Part of the research was prepared while she was a visiting scholar at ETS in Princeton, NJ, which was funded by a travel grant from the Fund for Scientific Research of Flanders (Belgium). The latter organization also funded the second author as a research assistant. The construction of the test used in the example was funded by the OBPWO grant 93.05 of the Ministry of the Flemish Community, Department of Education, awarded to Paul De Boeck and Frans Daems. The third author worked as a staff member on this project and also on the project G.0207.97 of the Belgian Fund for Scientific Research awarded to Paul De Boeck and Iven Van Mechelen. We would like to thank Isaac Bejar, Andrew Gelman, Eric Maris, Gert Storms, and Tom Verguts for helpful discussions and useful suggestions. Finally, we acknowledge the F/96/6 fellowship to Andrew Gelman and the OT/96/10 project of the University of Leuven. 
mastery (Meskauskas, 1976) implied that students can be ordered along a continuously-distributed ability dimension. In line with the continuum view of mastery it can also be expected that the items corresponding to learning tasks of a certain domain can be ordered along the same continuum as a function of their difficulty. Hence, when a cutoff point on the continuum is needed for educational decision-making on mastery two approaches are possible. In an examineecentered approach, the cutoff is determined as a function of the position along the continuum of a selected group of students, who are judged to be in a mastery, nonmastery, or borderline category. In an item-centered approach, the cutoff is determined as a function of the position along the continuum of a selected group of items, for instance, items that experts think masters of a domain should be able to solve correctly.

The distinction between an examinee-centered and an item-centered approach was originally proposed to categorize the numerous standard-setting procedures for criterion-referenced measurement within a continuum view on mastery (for reviews and discussions of these procedures see, e.g., Berk, 1986, 1996; Cizek, 1993, 1996a, b; Hambleton, 1980, 1982; Jaeger, 1989; Kane, 1994; Livingston \& Zieky, 1982; Norcini \& Shea, 1997). These standard-setting procedures have been criticized a lot because they are based on the judgments of experts and do not provide consistent results both within and across different procedures: "The most consistent finding from the research literature on standard setting is that different methods lead to different results" (NAE, 1993, p. 24, cited in Berk, 1996). The reasons given for the lack of consistent results are manifold: The judgments would depend too heavily on the context of judgment and on the background of the judges (e.g., Chang, Dziuban, Hynes, \& Olson, 1996; Plake, Impara, \& Potenza, 1994, Plake \& Melican, 1989); the judgments about expected item performance would not be consistent with the item parameters (e.g., van der Linden, 1982); or some judgments would be too complex a cognitive task (Shepard, 1995, cited in Crocker \& Zieky, 1995). Partially in reply to these criticisms, the recent literature on standard setting has devoted much attention to the justification and correct execution of the different phases in a standard setting study (Berk, 1996; Cizek, 1993, 1996b; Norcini \& Shea, 1997). Note that neither the examinee- nor the item-center approach takes into account possible costs of misclassification. A decision-theoretic approach to the problem of standard setting can be found in Huynh (1976), and Hambleton and Novick (1973), among others.

In contrast with a continuum view on mastery, a state view on mastery (Meskauskas, 1976) distinguishes only two levels of competence, mastery and nonmastery. The state view assumes learning to be an all-or-none process, with mastery corresponding to the presence of ability. This view implicitly demands that masters answer all items correctly and that nonmasters answer all items incorrectly. However, in applications of the state view in criterion-referenced measurement, it is acknowledged that a $100 \%$ performance standard is unrealistic. In what Glass (1978) called the "counting backwards from 100\%" method, 
judges rate the percentage of mistakes allowed by masters. In the application of latent class analysis to distinguish masters from nonmasters (see, e.g., Bergan \& Stone, 1985; Macready \& Dayton, 1980), deviations from the perfect and totally imperfect response patterns are explained as omission errors (false nonmasters) and intrusion errors (false masters). Note that in a latent class model, the classification of respondents is based on a psychometric model that is estimated on the basis of the actual performance of the respondents on the test.

The standard-setting methods based on the state view of mastery have not been used often in criterion-referenced measurement. The main critique of the state view (see Berk, 1980; Jaeger, 1989) is that its assumptions are unrealistic. First, the state view would not represent the heterogeneity of the population of respondents adequately. Second, because of the assumption that mastery is an all-or-none quality, the view only seems to be appropriate for highly homogeneous domains, like low-level cognitive skill, or very restricted factual knowledge. Finally, some of the methods based on the state view are restricted to tests with a small number of items.

The purpose of the present article is to propose a new model for mastery classification. The model is based on the continuum view of mastery and takes an item-centered approach to the determination of cutoff points. In line with some of the standard-setting procedures based on the state view, the classification of students is based on a psychometric model, so that it depends to a lesser extent on the judgment of experts.

The new model combines the framework of item response theory (IRT) with a multilevel formulation on the item side. This results in a hierarchical IRT model for criterion-referenced measurement. The model is based on the two-parameter IRT model, which is used to estimate the position of the persons and the items along a unidimensional latent continuum. The multilevel component of the model consists in the fact that items are grouped under criteria. To each criterion corresponds a group of items. This group of items, and, hence, the criterion itself, is characterized by a difficulty and discrimination parameter that are measured on the same latent scale as the person and item parameters. Hence, the position of the persons on the latent scale can be compared directly with the position of the criterion. The level of proficiency of a person with respect to the criterion is defined as the subject's probability of success on that criterion. Masters of the criterion are characterized by a high probability of success, and nonmasters by a low probability. Classification into those two classes is based on a cutoff point of .50 on the probability scale.

In the following, a formal presentation of the hierarchical IRT model for standard setting is given first. In the next two sections, the estimation and checking of the model are explained. The hierarchical IRT model is estimated using the Gibbs sampler combined with the technique of data augmentation. Other examples of the use of this Bayesian technique for the estimation of IRT models can be found in Patz (1996) and Bradlow, Wainer, and Wang (1996). The model checking for the hierarchical IRT model is based on the technique of 
posterior predictive checks. The application of the model is illustrated with a test measuring the attainment targets of reading comprehension in Dutch for children at the end of primary education.

\section{Model \\ The Hierarchical IRT Model}

Consider a criterion-referenced test measuring $K$ criteria (with $K \geq 1$ ) that are each operationalized with $I_{k}(k=1, \ldots K)$ items. Each item $i\left(i=1, \ldots I_{k}\right)$ measures a single criterion $k$ and is scored dichotomously. The probability that a person $v(v=1, \ldots, n)$ answers item $i$ of criterion $k$ correctly (i.e., $X_{v i k}=1$ ), is modeled according to the two-parameter normal ogive item response model (Lord \& Novick, 1968):

$$
\operatorname{Pr}\left(X_{v i k}=1\right)=\Phi\left(\alpha_{i k}\left(\theta_{v}-\beta_{i k}\right)\right),
$$

where $\Phi($.$) denotes the cumulative standard normal distribution, \alpha_{i k}$ the item discrimination parameter, $\theta_{v}$ the person ability parameter, and $\beta_{i k}$ the item difficulty parameter.

It is assumed that each criterion $k$ is characterized by a certain difficulty $\xi_{k}$ on the latent scale and by a power $\omega_{k}$ to discriminate among the respondents on the scale. The difficulty parameters of the items measuring a certain criterion are assumed to be located around the mean $\xi_{k}$ with variance $\sigma^{2}$ :

$$
\mathrm{N}\left(\beta_{i k} ; \xi_{k}, \sigma^{2}\right),
$$

where $\mathrm{N}$ denotes the normal distribution. Likewise, the item discrimination parameters of the items belonging to criterion $k$ are assumed to be located around $\omega_{k}$ with variance $v^{2}$ :

$$
\mathrm{N}\left(\alpha_{i k} ; \omega_{k}, v^{2}\right) .
$$

Note that the variance parameters $\sigma^{2}$ and $v^{2}$ can in principle also be made criterion-specific. They were chosen to be constant across criteria because the number of items measuring each criterion is sometimes too small to get a stable estimate of a variance parameter, as in the application we had in mind. Note also that Equations 2 and 3 can be considered as formalizations of the basic assumption in generalizability theory that the items of a test are a sample from a certain universe of items.

Combining Equations 1 to 3 leads to an IRT model with a hierarchical structure on the item side. The parameters $\xi_{k}, \omega_{k} \sigma^{2}$, and $v^{2}$ are the so-called hyperparameters of the model. They give a probabilistic specification of the item parameters, $\alpha_{i k}$ and $\beta_{i k}$, with which the observable outcomes are modeled. A graphical outline of the model is presented in Figure 1 by means of a directed acrylic graph (DAG, see, e.g., Spiegelhalter, Best, Gilks, \& Inskip, 1996). In a DAG, circles denote unobserved variables and squares observed variables. The 


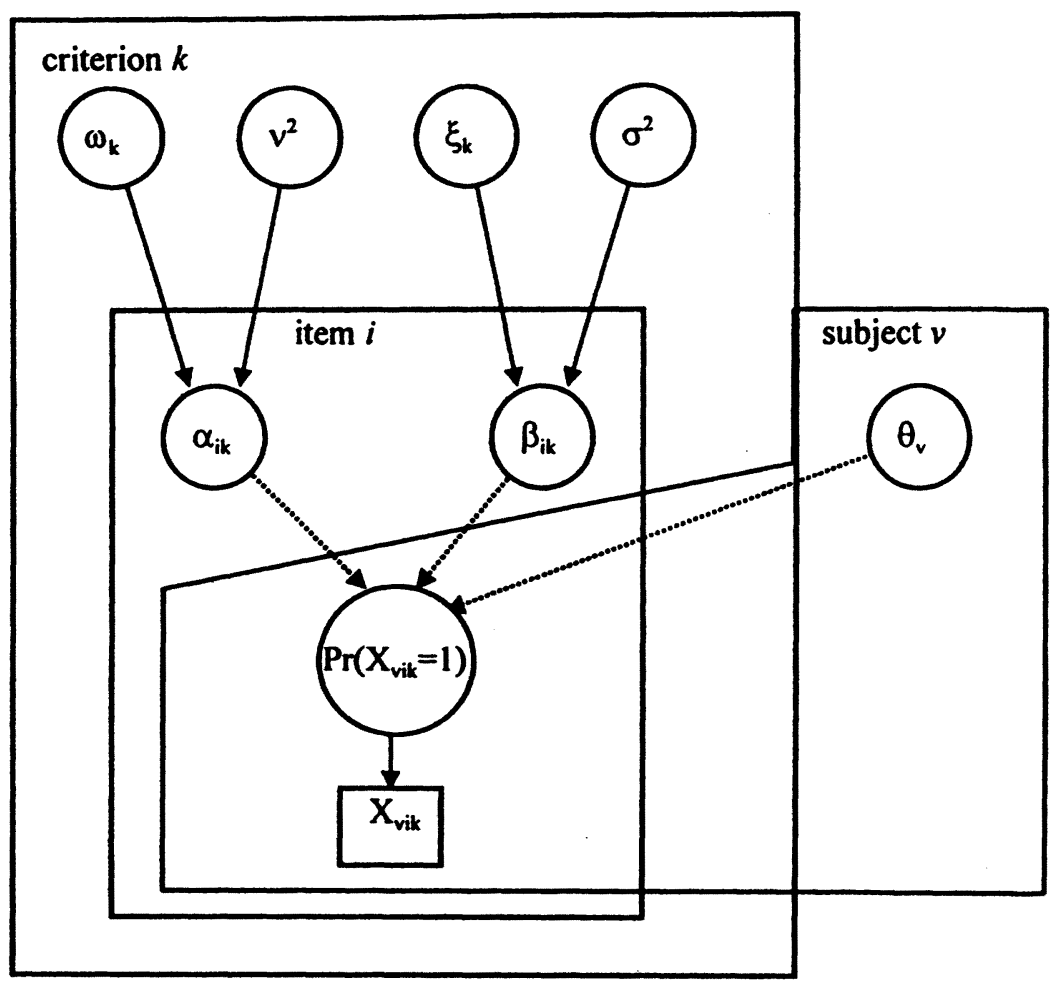

FIGURE 1. Graphical representation of the hierarchical IRT model

solid arrows refer to probabilistic relations, whereas the dotted arrows correspond to the deterministic relations in the model. The variables that are captured in the same polygon characterize the same real-world component. It can be seen in Figure 1 that person and item characteristics determine the probability of success on a certain item (cf. Equation 1). The items are nested within a criterion and the item characteristics are a function of the hyperparameters of the model. The figure clarifies the hierarchical structure of the model, but it leaves the distributional forms of the parameters unspecified.

\section{Its Use in Mastery Classification}

In the hierarchical IRT model a criterion is measured on the same scale as the person ability parameters. Hence, given the parameter values, one can calculate the probability of mastering criterion $k$ for each respondent $v$ by assuming that the criterion acts like an unobserved "superitem" $Y_{v k}$ that has to be solved correctly $\left(Y_{v k}=1\right)$ :

$$
\operatorname{Pr}\left(Y_{v k}=1\right)=\Phi\left(\omega_{k}\left(\theta_{v}-\xi_{k}\right)\right)
$$


Using a set of cutoff points on the probability scale, respondents can, for example, be classified into three categories with respect to each criterion: nonmastery (probability $\operatorname{Pr}<0.5)$, transition stage $(0.5 \leq \operatorname{Pr} \leq 0.8)$, and mastery $(\operatorname{Pr}>0.8)$. When determining these probabilities, and hence, when classifying respondents, the uncertainty about the estimated parameter values should be taken into account, that is, the uncertainty about the difficulty and discriminatory power of the superitem, and the uncertainty about the ability parameters. In the example given below, one way of dealing with the uncertainty about the estimate parameters follows from our Bayesian approach to model estimation and checking, as will be shown.

\section{Estimation}

\section{A Bayesian Framework}

A Bayesian approach (see, e.g., Gelman, Carlin, Stern, \& Rubin, 1995) was taken for the estimation of the hierarchical IRT model. This implies that the model has to be supplemented with prior distributions for certain parameters. The parameters in the hierarchical IRT model that need prior distributions are those in Figure 1 that do not have 'parent nodes' in the graph, i.e., those parameters that are not the endpoints of at least one arrow. Hence, the hyperparameters and the person ability parameter need a prior distribution. For $\xi_{k}$ and $\omega_{k}$ $(k=1, \ldots, K)$, non-informative priors were chosen. As prior distributions for the variance parameters $\sigma^{2}$ and $v^{2}$, inverse chi-square distributions with one degree of freedom were chosen, because this distribution is mathematically convenient for variance parameters in normal models (Gelman et al., 1995). Finally, the prior for the person ability parameters $\theta_{v}$ was a standard normal distribution. Note that fixing the mean and variance of the prior for the person ability distribution is necessary to make the IRT model identifiable with respect to the location and scale of the latent dimension.

Given these prior distributions, the posterior distribution of the parameters given the data becomes

$$
\begin{aligned}
& p\left(\alpha, \beta, \theta, \xi, \sigma^{2}, \omega, v^{2} \mid \mathbf{x}\right) \propto p\left(\sigma^{2}\right) p\left(v^{2}\right)\left(\prod_{k=1}^{K} \prod_{i=1}^{\mathrm{I}_{k}} \mathrm{~N}\left(\beta_{i k} ; \xi_{k}, \sigma^{2}\right)\right)\left(\prod_{k=1}^{K} \prod_{i=1}^{\mathrm{I}_{k}} \mathrm{~N}\left(\alpha_{i k} ; \omega_{k}, v^{2}\right)\right) \times \\
& \left(\prod_{v=1}^{n} \mathrm{~N}\left(\theta_{v} ; 0,1\right)\right)\left(\prod_{v=1}^{n} \prod_{k=1}^{K} \prod_{i=1}^{\mathrm{I}_{k}}\left(\operatorname{Pr}\left(X_{v i k}=1\right)\right)^{x_{i i k}}\left(1-\operatorname{Pr}\left(X_{v i k}=1\right)\right)^{I-x_{v i k}}\right) .
\end{aligned}
$$

Looking at the marginal posterior distribution for each model parameter gives an idea of the posterior uncertainty of the parameter at hand, given the data and the prior distributions. The mean (or mode) of the marginal posterior distribution can be used to summarize the distribution. The standard deviation of the marginal posterior distribution of a parameter gives an indication of the standard error of estimation. 


\section{The Gibbs Sampler}

In order to obtain a sample from the posterior in Equation 5, we applied the Gibbs sampler, which is an iterative technique that belongs to the class of Markov Chain Monte Carlo (MCMC) techniques (Gelman et al., 1995; Gilks, Richardson, \& Spiegelhalter, 1996; Tanner, 1996). Without going into details, the Gibbs sampler can be described as a modular approach to sampling from the posterior distribution, creating a Markov chain by successively sampling from the set of the full conditional distributions, so that once this Markov chain has converged to its equilibrium distribution, one samples from the posterior distribution.

A crucial step is the derivation of the conditional distributions. Albert (1992) showed for standard normal ogive IRT models that these conditional distributions can be derived analytically when the posterior distribution of the IRT model is augmented with latent data (Tanner, 1996). The data augmentation component in Albert's method consists in introducing a normally distributed latent variable $Z_{v i k}$ with a mean that is a function of the person and item parameters:

$$
\mathrm{N}\left(Z_{v i k} ; \alpha_{i k}\left(\theta_{v}-\beta_{i k}\right), 1\right) \text {. }
$$

It is imposed that $Z_{v i k} \leq 0$ when $X_{v i k}=0$ and that $Z_{v i k}>0$ when $X_{v i k}=1$. Hence, the probability that $Z_{v i k}>0$ equals the probability of a correct response as given by Equation 1. Applying the data augmentation method to the posterior given in Equation 5 results in the following augmented posterior distribution for the hierarchical IRT model:

$$
\begin{aligned}
& p\left(\alpha, \beta, \theta, \mathbf{Z}, \xi, \sigma^{2}, \omega, v^{2} \mid \mathbf{x}\right) \propto p\left(\sigma^{2}\right) p\left(v^{2}\right) \\
& \times\left(\prod_{k=1}^{K} \prod_{i=1}^{I_{k}} \mathrm{~N}\left(\beta_{i k} ; \xi_{k}, \sigma^{2}\right)\right)\left(\prod_{k=1}^{K} \prod_{i=1}^{I_{k}} \mathrm{~N}\left(\alpha_{i k} ; \omega_{k}, v^{2}\right)\right)\left(\prod_{v=1}^{n} \mathrm{~N}\left(\theta_{v} ; 0,1\right)\right) \\
& \times\left(\prod_{v=1}^{n} \prod_{k=1}^{K} \prod_{i=1}^{I_{k}} \mathrm{~N}\left(Z_{v i k} ; \alpha_{i k}\left(\theta_{v}-\beta_{i k}\right), 1\right)\left(\mathrm{I}\left(Z_{v i k}>0\right) \mathrm{I}\left(x_{v i k}=1\right)+\mathrm{I}\left(Z_{v i k} \leq 0\right) \mathrm{I}\left(x_{v i k}=0\right)\right)\right),
\end{aligned}
$$

where $\mathrm{I}($.$) is the indicator function.$

The conditional distributions for the Gibbs sampler are given in the Appendix. They all are either a truncated normal, a normal, or a scaled inverse chi-square distribution, from which one can sample directly using standard routines. Note that, due to local independence, a lot of parameters cancel out when calculating a conditional distribution for a particular parameter from the joint augmented posterior distribution in Equation 7. The mathematical tractability of the conditional distributions is not only due to the data augmentation step, but also to the form of the priors and for the hierarchical model assumption (Equations 2 and 3).

\section{Convergence of the Markov Chain}

A critical issue in applying MCMC techniques is the question how long the Markov chain must be in order to be confident that it has converged, and hence, that one actually samples from the posterior distribution. There is currently no 
general agreement on a method for determining convergence of the Gibbs sampler (see, e.g., Gelman \& Rubin, 1992; Zellner \& Min, 1995). In the present paper, two criteria of convergence are used. First, for each model parameter the convergence measure $\sqrt{\hat{R}}$ proposed by Gelman and Rubin (1992) is calculated. This measure is based on the idea that chains, running from different starting points in the parameter space, have reached convergence at the point where the variance of the sampled parameter values between the chains approximates the variance of the sampled parameter values within the chains. Formally, Gelman and Rubin (1992) developed the measure $\hat{R}$, which quantifies the estimated ratio of between-chain variation to within-chain variation of a single parameter. If one demands the MCMC algorithm to be converged, $\sqrt{\hat{R}}$ has to be definitely smaller than 1.1 for all parameters of the model. Second, for different batches of consecutive draws from the posterior, the posterior mean and posterior standard deviation were calculated for each parameter (Hoijtink \& Molenaar, 1997). Small differences among the batches indicate that the Gibbs sampler has converged.

\section{Goodness of Recovery}

A small goodness-of-recovery study was performed for the hierarchical IRT model. In this study, the number of items measuring each criterion and the within-criterion variance $\sigma^{2}$ of the item difficulty parameters were manipulated. For each simulated data set, the responses of 500 subjects were simulated to either 15 or 30 items measuring three criteria on one dimension. The data were estimated according to the hierarchical IRT model and according to the twoparameter IRT model. The latter model was estimated with the program BIMAIN (Zimowski, Muraki, Mislevy \& Bock, 1994), which uses a marginal maximum likelihood procedure and maximum a posteriori estimates for the ability parameters.

In general, the goodness of recovery was satisfactory. The most important findings were the following. First, the stimulated item and person parameters of the hierarchical IRT model correlated almost perfectly with the corresponding parameters from the two-parameter IRT model as estimated by BIMAIN. Second, the goodness of recovery was very good for the item difficulty parameters (correlations around .98), reasonably good for the person ability parameters given the small number of items (correlations around .85), but not so good for the item discrimination parameter (correlations around .60). The latter result was probably due to the limited number of persons in the test, which may not have been sufficient to get a reliable estimation of this parameter (neither with BIMAIN nor with the Gibbs sampler). Finally, with respect to the hyperparameters, it was found that recovery of the hyperparameters depended on the number of items (the higher, the better recovery), and, for the $\xi_{k}$, also on the withincriterion variance of the item difficulty parameters (the smaller the variance, the better recovery). The results are all in line with what one would expect. Despite their obviousness, the results give a verification of the estimation procedure. 


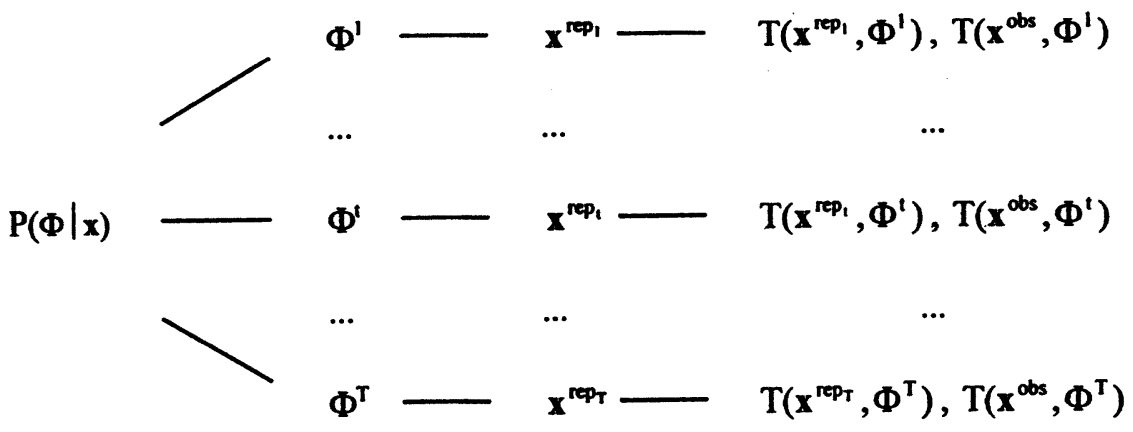

FIGURE 2. Graphical outline of the calculation of a PPC

\section{Model Checking \\ Posterior Predictive Checks}

Within Bayesian data analysis, whether or not a model fits the data can be determined by using the technique of Posterior Predictive Checks (PPC, see Gelman et al., 1995; Gelman \& Meng, 1996; Gelman, Meng, \& Stern, 1996; Meng, 1994; and Rubin, 1984). The basic idea of a PPC is that "if the model fits, then replicated data generated under the model should look similar to observed data" (Gelman et al., 1995, p. 165). The fit of the model is assessed by defining a test quantity that measures the discrepancy of the data to some aspects of the model. Variation of the test quantity for the replicated data denotes the random fluctuation of the test quantity under the model's own assumptions. If the model does not fit the data, the test quantity should be systematically higher when calculated on the data than when calculated on the replicated data generated under the model. A PPC $p$-value for assessing model fit is defined as

$$
\text { PPC } p \text {-value }=1-\operatorname{Pr}\left[\mathrm{T}(x, \Phi)>\mathrm{T}\left(x^{\text {rep }}, \Phi\right) \mid x\right] \text {, }
$$

where $\Phi$ indicates the parameter vector $\left(\phi_{1}, \ldots, \phi_{r}\right), \mathrm{T}(x, \Phi)$ is the test quantity that may depend on the model parameters and that is calculated on the data, and $\mathrm{T}\left(x^{\text {rep }}, \Phi\right)$ is the same test quantity, but calculated on the replicated data. Small values of the PPC $p$-value indicate bad fit. Note that when using a PPC, researchers are free to develop their own test quantities, checking different aspects of the model. In contrast with classical test statistics, Bayesian test quantities are not restricted to test quantities with theoretically derived sampling distributions, and they also allow for dependence of the test quantities on the model parameters.

Figure 2 gives a graphical outline of the calculation of a PPC in practice. For each draw $\Phi^{t}$ of parameter values from the posterior distribution, one generates replicated data for which one calculates the test quantity. If the test quantity 
depends on both model and data, the test quantity is also calculated for the observed data using the same draw $\Phi^{t}$ of parameter values. If the test statistic does not depend on the model parameters $\Phi, \mathrm{T}(x, \Phi)$ simplifies to $\mathrm{T}(x)$ and has to be calculated only once. The extremity of the test quantity for the observed data in comparison with the test quantity for the replicated data is assessed by the PPC $p$-value. The PPC $p$-value is estimated by the proportion of draws of the posterior for which the inequality in the right-hand side of Equation 8 holds.

\section{Global Goodness of Fit for the Hierarchical IRT Model}

As a measure of global goodness of fit for the hierarchical IRT model, a chi-square statistic was chosen. This test quantity has proven its usefulness in testing IRT models within the framework of maximum likelihood procedures (see, e.g., Glas, 1988). The chi-square statistic compares the observed number of correct and incorrect responses to the expected number of correct and incorrect responses in different ability groups on each item. It can be calculated as follows for each item $i$ :

$$
\chi_{i}^{2}=\sum_{g=1}^{G} \frac{n_{g i}\left(p_{g i}-\pi_{g i}\right)^{2}}{\pi_{g i}\left(1-\pi_{g i}\right)},
$$

where $n_{g i}$ indicates the number of persons in ability group $g, p_{g i}$ the proportion of correct answers, and $\pi_{g i}$ the expected proportion of correct answers in ability group $g$. The chi-square statistic is summed over items, yielding a discrepancy measure for the test as a whole. When used as a test quantity for a PPC, the chi-square statistic has to be calculated for each draw from the posterior, as $\pi_{g i}$ depends on the model parameters. We formed $G$ adjacent and non-overlapping ability groups on the basis of the sorted vector of the person ability parameters from that draw. The $\pi_{g i}$ are calculated as the mean probability of a correct response for all the persons in the ability group. The chi-square statistic is calculated twice for each draw of parameters: once with the $p_{g i}$ based on the replicated data and once with the $p_{g i}$ based on the observed data. The values of the chi-square statistics for all draws from the posterior are plotted in a scatter diagram. The proportion of draws from the posterior for which the chi-square statistic is higher for the observed data than for the replicated data is calculated to estimate the PPC $p$-value of the model.

Replicated data can be generated fairly easily in the hierarchical IRT model by first calculating the probability of a correct response (see Equation 1) for each set of drawn item and person parameters $\alpha_{i k}, \beta_{i k}$, and $\theta_{v}$ from the posterior. This probability is then dichotomized by comparing it to a random draw $u$ from the uniform distribution on the interval $[0,1]$. The replicated value of $x_{v i k}^{\text {rep }}$ is set to 1 if the calculated probability of a correct response exceeds $u$, and to 0 otherwise.

\section{Robustness and Sensitivity Analysis}

Even when a model provides good fit for the observed data, posterior inferences can be highly sensitive to the distributional assumptions of the model. In a 
TABLE 1

Text Type, Level of Processing, and Number of Items for the Attainment Targets of the Test

\begin{tabular}{llll}
\hline & & Level of & \\
$k$ & Text Type & Processing & $I_{k}$ \\
\hline 1 & instructions & retrieving & 4 \\
2 & articles in magazine & retrieving & 6 \\
3 & study material & structuring & 8 \\
4 & tasks in textbook & structuring & 5 \\
5 & comics & structuring & 9 \\
6 & stories, novels & structuring & 6 \\
7 & poems & structuring & 8 \\
8 & newspapers for children, textbooks, encyclopedias & evaluating & 6 \\
9 & advertising material & evaluating & 5 \\
\hline
\end{tabular}

robustness and sensitivity analysis different distributions for likelihood and priors are applied in order to determine whether the posterior inferences vary in comparison with the original model. For the hierarchical IRT model, the assumption of a normal distribution for the person parameter $\theta_{v}$ and for the item parameters $\alpha_{i k}$ and $\beta_{i k}$ is investigated by applying a $t$-distribution with varying degrees of freedom in place of the normal distribution. A $t$-distribution has a longer tail than a normal distribution and is therefore less sensitive to the effect of possible outliers.

\section{Example}

\section{A Flemish Test for Reading Comprehension}

The Parliament of the Flemish Community in Belgium recently issued a set of attainment targets specifying the expected basic competencies of children who leave primary education. The Department of Education funded a project (see De Boeck, Daems, Meulders, \& Rymenams, 1997) to devise a test for assessing the attainment targets of reading comprehension in Dutch (being the official language in Flanders). These attainment targets were conceptualized as the ability to process information in texts that are meaningful to children. Each attainment target was characterized by two factors: (1) the required level of processing and (2) the type of text. Three levels of processing were distinguished, namely, retrieving, structuring and evaluating information in a text. A variety of texts were specified. We focus on a test for the first nine attainment targets. Table 1 gives a description of the contents of the test and of the number of items that were used to measure each attainment target. It was assumed that the three ways of processing (and, hence, the nine attainment targets) refer to a common underlying reading ability dimension. However, it should be clear that this unidimensionality with respect to the latent continuum does not imply that all attainment targets are equal in difficulty. Hence, some pupils may master one 
attainment target while not being able to solve the items of a more difficult target. The ordering of the attainment targets on the basis of their difficulty may provide relevant information regarding the order in which these attainment targets should be taught in the curriculum.

\section{Method}

The fourth author of the test (R. Rymenams) and school teachers collaborated in compiling the test (choosing texts and items). The items were of mixed format. Of the 57 items measuring the 9 attainment targets, 22 used a multiplechoice format and 35 an open format. All items were scored dichotomously.

The test was administered to 539 children who were in the final year of primary education. Note that the data were actually gathered at the beginning of the school year, while the attainment targets specify what pupils should master only at the end of primary school. The timing of the administration of the test was less important for the original project as the goal was test construction and not educational assessment of the actually participating children.

\section{Estimation}

Check of unidimensionality. As an initial check of the unidimensionality of the items, a full-information item factor analysis was performed on the data using TESTFACT (Wilson, Wood, \& Gibbons, 1991). The eigenvalues of the first six factors were 11.0, 2.1, 2.0, 1.9, 1.9, and 1.8, indicating that a unidimensional solution seems appropriate.

Convergence of the Markov chain. Ten Markov chains were run using random starting points for the item and person parameters. For each $\theta_{v}$ and each $\beta_{i k}$ a starting value was sampled from the standard normal distribution. For $\alpha_{i k}$ a starting value was sampled from the uniform distribution with a minimum of 0 and a maximum of 2 . The starting points for the hyperparameters consisted in the mean and variance of the starting values for the item difficulty and item discrimination parameters.

Each chain was run for 2000 iterations. The first 500 iterations of each chain were discarded. The remaining 1500 iterations were used to calculate the convergence measure $\sqrt{\hat{R}}$. It varied between 1.00 and 1.06 with a median of 1.00 for all the parameters of the model. There were no systematic differences in convergence among the parameters, for example, between the hyperparameters and the other parameters. The last 1500 iterations of the 10 chains were also divided into 30 batches of 500 subsequent draws from the posterior distribution. The means and standard deviations of each parameter calculated in those 30 batches were very similar for all parameters. Hence, one can be confident that the Gibbs sampler reached convergence.

For the estimation and checking of the model, every fifth draw in the remaining 1500 draws of each of the 10 chains was accepted in order to have less 
TABLE 2

Results for the Hyperparameters $\xi_{\mathrm{k}}$ and $\omega_{\mathrm{k}}$

\begin{tabular}{ccrcrcccccc}
\hline & & \multicolumn{3}{c}{$\xi_{k}$} & \multicolumn{2}{c}{ Mean $\beta_{i k}$} & \multicolumn{2}{c}{$\omega_{k}$} & \multicolumn{2}{c}{ Mean $\alpha_{i k}$} \\
\cline { 2 - 10 }$k$ & $I_{k}$ & Mean & $S D$ & Mean & $S D$ & Mean & $S D$ & Mean & $S D$ \\
\hline 1 & 4 & -0.89 & 0.86 & -0.89 & 0.11 & 0.98 & 0.16 & 0.98 & 0.07 \\
2 & 6 & -1.13 & 0.74 & -1.13 & 0.11 & 0.91 & 0.14 & 0.91 & 0.06 \\
3 & 8 & -1.23 & 0.62 & -1.23 & 0.14 & 0.76 & 0.12 & 0.76 & 0.05 \\
4 & 5 & 0.06 & 0.80 & 0.06 & 0.22 & 0.74 & 0.14 & 0.74 & 0.06 \\
5 & 9 & -1.41 & 0.59 & -1.42 & 0.15 & 0.71 & 0.11 & 0.72 & 0.05 \\
6 & 6 & -0.66 & 0.73 & -0.67 & 0.13 & 0.80 & 0.14 & 0.80 & 0.06 \\
7 & 8 & -1.09 & 0.63 & -1.09 & 0.15 & 0.79 & 0.12 & 0.79 & 0.05 \\
8 & 6 & 0.57 & 0.71 & 0.59 & 0.11 & 0.82 & 0.14 & 0.82 & 0.06 \\
9 & 5 & -2.44 & 0.84 & -2.44 & 0.34 & 0.54 & 0.14 & 0.55 & 0.05 \\
\hline
\end{tabular}

Note. The parameter estimates are given on a logistic scale; i.e., in Equation 1, a scale factor $\mathrm{S}=1.7^{-1}$ has to be inserted.

dependence between subsequent draws from the Gibbs sampler. This results in a total sample of 3000 draws from the augmented posterior on which the parameter estimation and model checking was based.

Parameter estimation. The posterior means for the item difficulty parameters $\beta_{i k}$ varied between -4.89 and 4.64 with a mean of -.95 . Given that the origin of the scale was fixed at the mean of the prior distribution for the person ability parameters, this result implies that most of the items were relatively easy for the respondents. The posterior means of the person ability parameters $\theta_{v}$ varied between -2.63 and 2.23 with a mean of -.001 . The posterior means of the item discrimination parameters $\alpha_{i k}$ varied between .29 and 1.30 with a mean of .78 .

Table 2 gives a summary of the estimates for the hyperparameters $\xi_{k}$ and $\omega_{k}$. The posterior means of the marginal posterior distribution of $\xi_{k}$ are given in the third column. Attainment target 9 was the easiest and attainment target 8 the most difficult. The posterior means of the hyperparameters $\xi_{k}$ were almost the same as the posterior means of the item difficulty parameters $\beta_{i k}$ measuring the same attainment target (see column 5). However, the standard deviations around the latter means were much smaller than the posterior standard deviations of the $\xi_{k}$. The posterior standard deviations of $\xi_{k}$ were smaller for attainment targets with more items. In contrast, there was no clear relationship between the standard deviations of the mean $\beta_{i k}$ parameters and the number of items measuring the attainment target.

For the discrimination powers $\omega_{k}$ of the attainment targets, it was found that eight of the nine posterior means varied between .71 and .98. For attainment target 9 a relatively low value was found. The standard deviation of the posterior of the $\omega_{k}$ was almost linearly related to the number of items measuring the attainment target, as was the case for the $\xi_{k}$. The posterior means of $\omega_{k}$ were 
almost equal to the posterior means of the $\alpha_{i k}$ measuring the attainment target $k$. Again, the standard deviations of the latter means were smaller than the standard deviations of the estimated hyperparameters.

The posterior mean of the variance $\sigma^{2}$ among the item difficulties within attainment targets was 3.02 with a posterior standard deviation of .73. This relatively high mean variance indicates that there was a high overlap among the attainment targets in the distribution of the item difficulty parameters of the items measuring each attainment target. For the variance $v^{2}$ among the discrimination parameters, the posterior mean was .09 with a posterior standard deviation of .02 .

\section{Model Checking}

Posterior Predictive Checks. For the calculation of the chi-square goodness-offit measure, eight ability groups were formed with as boundary scores the .125 quantiles of the vector of person ability parameters of each draw from the posterior. The estimated PPC $p$-values for the chi-square statistics on item level indicated good model fit. Their values varied between .05 and .58 with a median of .51 . The one item with a $p$-value of .05 was the item with the highest item difficulty parameter (namely 4.64) and with almost no differentiation among the respondents that took the test. It is important to note that no consistent differences in fit occurred between the items with a multiple-choice format and those with an open format. This indicates that for the modeling of the response behavior on the multiple-choice items no guessing factor (and, hence, no threeparameter IRT model) was needed.

The chi-square values were summed over the items of the test for every draw from the posterior distribution, yielding a global measure of fit at the test level. A plot of the total chi-square values for the replicated data and for the observed data is given in Figure 3 for the 3000 draws from the posterior distribution. The PPC $p$-value is .21, indicating that the model fits the data relatively well. When the item with the $p$-value of .05 is eliminated from the test, the PPC $p$-value for the total chi-square statistic equals .30 .

Robustness and Sensitivity Analysis. The effect of the assumption of a normal distribution for $\theta_{v}, \alpha_{i k}$, and $\beta_{i k}$ was investigated by changing the normal distribution into a $t$-distribution with degrees of freedom equal to $1,2,4,5,10$, or 30 . Posterior inferences for each parameter under the $t$-distribution were computed using importance resampling (see, e.g., Gelman et al., 1995). For a sample of 5000 draws from the posterior, the importance ratios were calculated. This approximative method seemed well justified as the histogram of the logarithm of the importance ratios did not have a long tail. Then 500 draws from the posterior were resampled without replacement. The posterior means of these resampled values did not differ much from the posterior means obtained under the normal distribution. 


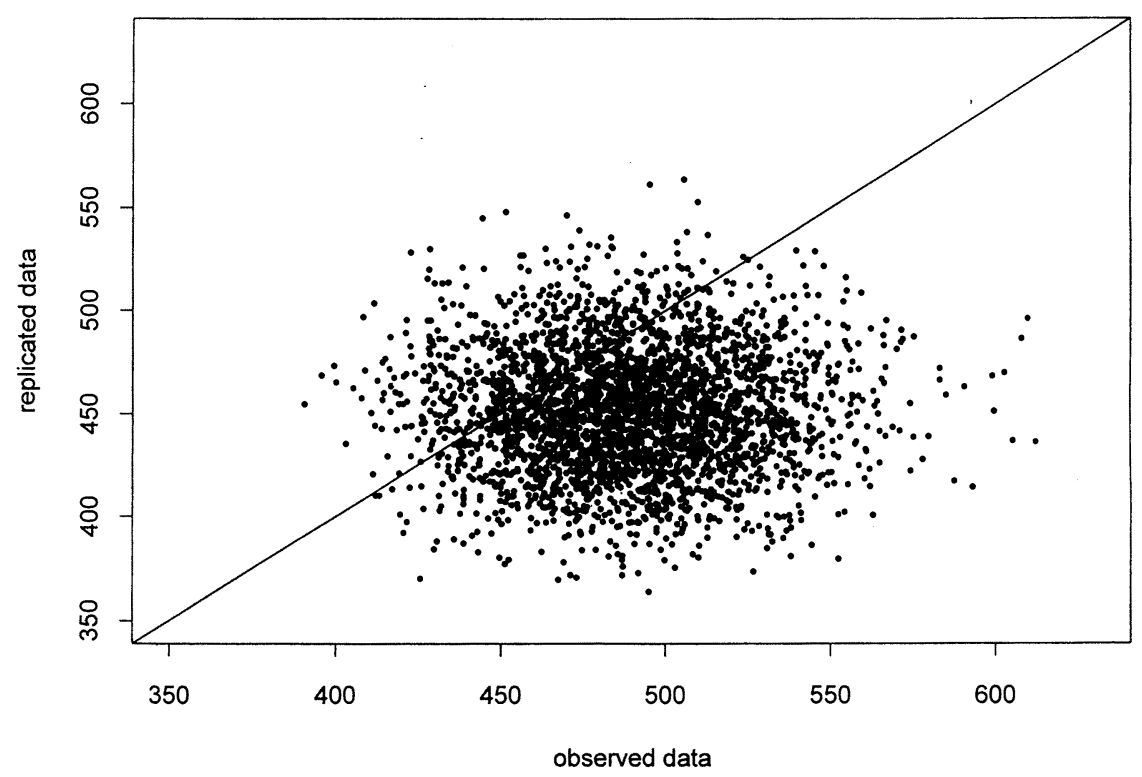

FIGURE 3. Chi-square values of the test for the observed versus the replicated data

\section{Application to Mastery Classification}

The probability that pupil $v$ masters attainment target $k$ can be computed according to Equation 4 for every draw of $\left(\theta_{v}, \xi_{k}, \omega_{k}\right)$ from the joint posterior. Note that the resulting distribution of the success probabilities takes into account the posterior uncertainty both with respect to the ability of pupil $v$ and with respect to the difficulty and the discriminating power of attainment target $k$. Figure 4 shows the distribution of the success probabilities for attainment target 4 for three pupils, with a low $\left(\hat{\theta}_{v}=-2.01\right)$, an intermediate $\left(\hat{\theta}_{v}=.54\right)$, and a high ability level $\left(\hat{\theta}_{v}=2.23\right)$, respectively, where the $\hat{\theta}_{v}$ are the estimated posterior means.

With the aim of classifying a pupil into the nonmastery, mastery, or transition category with regard to a specific attainment target, one needs to specify a rule that maps the distribution of success probabilities into these categories. We propose to count the number of times (over draws from the joint posterior) that the success probability of the pupil is in the nonmaster category $(\operatorname{Pr}<.05)$, in the transition category $(0.5 \leq P r \leq 0.8)$, or in the master category $(0.8<P r)$ and to assign the pupil to the category with the highest frequency. An overview of the decisions made in the sample of pupils could then be made by calculating the proportion of pupils in each category. However, this procedure does not give the best estimate of the proportion of pupils in the population that belong to each mastery category. To get an estimate of the population proportions, one should count the relative frequency of occurrence of the posterior probabili- 
Janssen, Tuerlinckx, Meulders, De Boeck
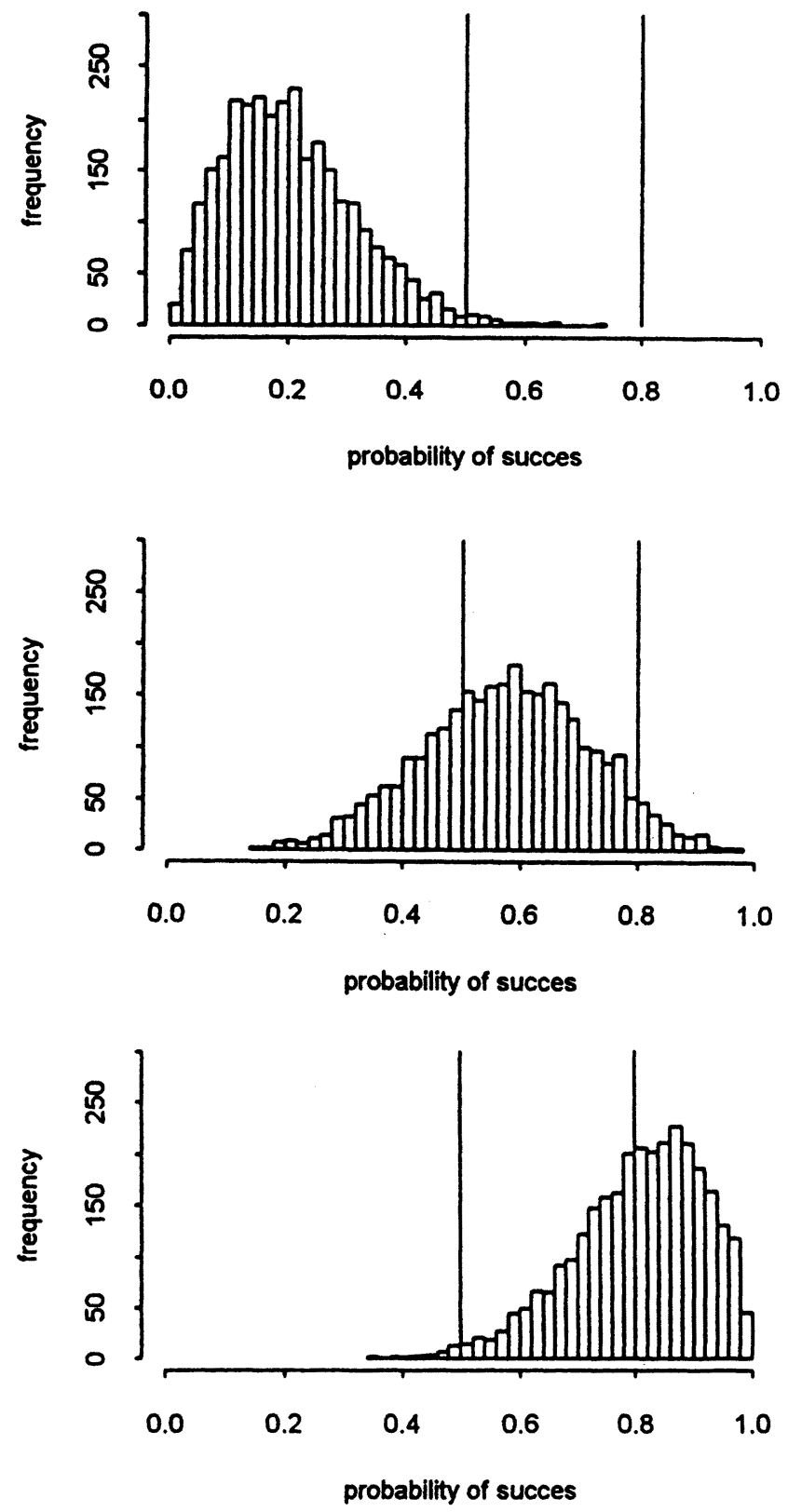

FIGURE 4. Histogram of the success probabilities on attainment target 4 for a pupil with a low (upper panel), an intermediate (middle panel), and a high ability (lower panel) (The bars denote the boundaries between the mastery classes.) 
TABLE 3

Estimated Percentage of Pupils in the Population in the Nonmastery, Transition, or Mastery Category for the Nine Attainment Targets

\begin{tabular}{cccc}
\hline$k$ & Nonmastery & Transition & Mastery \\
\hline 1 & 24.9 & 42.5 & 32.6 \\
2 & 18.4 & 46.0 & 35.6 \\
3 & 15.0 & 56.1 & 28.9 \\
4 & 51.4 & 42.7 & 5.9 \\
5 & 11.5 & 58.4 & 30.9 \\
6 & 29.6 & 52.4 & 18.0 \\
7 & 18.1 & 54.8 & 27.1 \\
8 & 67.5 & 29.9 & 2.6 \\
9 & 3.0 & 55.7 & 41.3 \\
\hline
\end{tabular}

ties $\operatorname{Pr}\left(Y_{v k}=1\right)$ in each master category over draws from the posterior and over the pupils in the sample. Table 3 gives the percentage of cases in which the $\operatorname{Pr}\left(Y_{v k}=1\right)$ were in each of the three mastery categories for the nine attainment targets. The percentages in Table 3 show some clear differences that are in line with the differences in difficulty among the attainment targets. The transition category was the modal category for seven out of nine attainment targets. For attainment targets 4 and 8 the nonmaster category was the modal category and only a small proportion of pupils in the population is estimated to be masters. Attainment target 9 (evaluating advertisements) showed the highest estimated proportion of masters.

\section{Discussion}

The hierarchical IRT model provides a model-based classification of respondents in case mastery decisions have to be made. In the hierarchical approach for determining the cutoff point, the criterion is considered as an unobserved, 'average' item. This approach is in line with judgmental procedures following an item-based approach for determining cutoff scores, as in the method of Angoff (1971) or Nedelsky (1954). In the latter procedures, judges rate the probability of success that is expected on every item from masters of the domain. The cutoff on the observed test score scale is then set at the sum of the expected performances on the items of the test. This approach parallels the idea of equating the criterion with a kind of average item, as is done in the hierarchical IRT model. Note that the hierarchical IRT model does not resolve the critique of Glass (1978) that any method leading to mastery classifications is in a sense arbitrary. As Shepard (1980) and others have noted, the respondents just below the cutoff score will not differ much in their ability from respondents just above the cutoff score.

An important requirement for the described approach to yield meaningful results is that the items selected for each criterion have content validity. The items should be a representative sample from the content domain of the test. 
Having content validity is a general requirement for criterion-referenced measures (e.g., Linn, 1980). The issue of content validity is a matter of judgment as well. However, methods like those proposed by Angoff (1970) and Nedelsky (1954) need probability of success judgments of each item in addition.

It was shown in the goodness-of-recovery study that the estimated item and person parameters of the hierarchical IRT model corresponded to the parameters estimated by a standard IRT model without a hierarchical structure imposed on the items. One could therefore wonder whether one could not use a standard IRT model and calculate the mean item difficulty for each criterion instead of estimating and testing the hierarchical IRT model. The answer is a general one: "[T]he main problem with substituting point estimates for the hyperparameters is that it ignores our real uncertainty about them" (Gelman et al., 1995, p. 141). This statement is in line with the finding that the mean of the item parameters almost equaled the hyperparameters, but that the posterior standard deviation around the mean item parameters was much smaller than the posterior standard deviation of the hyperparameters. Hence, when one has data with a multilevel structure, the uncertainty about the parameters of the higher levels should not be underestimated, as is the case when averaging point estimates at the lower level. Moreover, one can expect that the higher in the hierarchy, the less information one has about the parameters of that level. Therefore, the value of using hierarchical models will be eroded when the data contain an insufficient number of data points at the lowest level. For the hierarchical IRT model, the reliability of the estimated hyperparameters is directly related to the number of items measuring the criterion, as was shown in the goodness of recovery study and in the values of the posterior standard deviations for the hyperparameters in Table 2.

The hierarchical IRT model was developed for criterion-referenced measurement. Most criterion-referenced test measure a single criterion only, but in the given example a test measuring nine attainment targets of reading comprehension was analysed. It was shown that the attainment targets for reading comprehension set by the Flemish government are realistic educational objectives. Although it was not the aim of the study to assess the pupils, it was interesting to find that at the beginning of the final year of primary education, most of the pupils were in the transition class between nonmastery and mastery. The variability in the histograms of the success probabilities used for the mastery classification represents the uncertainty both with respect to the person ability parameter and the hyperparameters $\xi_{k}$ and $\omega_{k}$ of the criterion.

The Bayesian method for calculating the success probability takes into account the location of the estimated parameters together with their standard errors of measurement. This approach stands in sharp contrast to common practice in IRT to estimate the item parameters and to take them subsequently as true values for estimating examinees' proficiency parameters (see Mislevy, Wingersky, \& Sheehan, 1994, for another approach to this problem). Note that the posterior uncertainty for the person ability parameter is a function of its position on the latent scale: the more extreme the ability parameter in comparison to the loca- 
tion of the items, the higher its standard error of measurement. However, the posterior uncertainty regarding $\theta_{v}$ is also influenced by the person fit of the respondent. Hence, two respondents with the same posterior mean for $\theta_{v}$ can still have different posterior standard deviations.

The hierarchical IRT model assumes that the criteria define a partition of the items, implying that each item is used to measure only one criterion. However, in performance assessment, for example, so-called "integration items" are often constructed to provide scores in more than one content area. The modeling of such items would require an extension of the hierarchical IRT model, in which an item can contribute to the estimation of the hyperparameters of different criteria. This would correspond to a hierarchical formulation of the linear logistic test model of Fischer (1983), which is currently under investigation.

\section{Appendix}

The eight full conditional distributions of the Gibbs sampler are the following: $p\left(Z_{v i k} \mid \alpha_{i k}, \theta_{v}, \beta_{i k}, x_{v i k}\right)=\left\{\begin{array}{l}\mathrm{N}\left(Z_{v i k} ; \alpha_{i k}\left(\theta_{v}-\beta_{i k}\right), 1\right) \text { truncated at } 0 \text { to the left if } x_{v i k}=1 \\ \mathrm{~N}\left(Z_{v i k} ; \alpha_{i k}\left(\theta_{v}-\beta_{i k}\right), 1 \text { truncated at } 0 \text { to the right if } x_{v i k}=0\right.\end{array}\right.$

$$
\begin{aligned}
& \theta_{v} \mid Z_{v}, \beta, \alpha \sim \mathrm{N}\left(\frac{\sum_{k=1}^{K} \sum_{i=1}^{I_{k}} \alpha_{i k}\left(\mathrm{Z}_{v i k}+\alpha_{i k} \beta_{i k}\right)}{1+\sum_{k=1}^{K} \sum_{i=1}^{I_{k}} \alpha_{i k}^{2}}, \frac{1}{1+\sum_{k=1}^{K} \sum_{i=1}^{I_{k}} \alpha_{i k}^{2}}\right) \\
& \beta_{i k} \mid \theta, Z_{i k}, \alpha_{i k}, \xi_{k}, \sigma^{2} \sim \mathrm{N}\left(\frac{\alpha_{i k} \sum_{v=1}^{n}\left(\alpha_{i k} \theta_{v}-Z_{v i k}\right)+\frac{\xi_{k}}{\sigma^{2}}}{n \alpha_{i k}^{2}+\frac{1}{\sigma^{2}}}, \frac{1}{n \alpha_{i k}^{2}+\frac{1}{\sigma^{2}}}\right) \\
& \alpha_{i k} \mid \theta, Z_{i k}, \beta_{i k}, \omega_{k}, v^{2} \sim \mathrm{N}\left(\frac{\sum_{v=1}^{n}\left(\left(\theta_{v}-\beta_{i k}\right) Z_{v i k}\right)+\frac{\omega_{k}}{v^{2}}}{\sum_{v=1}^{n}\left(\theta_{v}-\beta_{i k}\right)^{2}+\frac{1}{v^{2}}}, \frac{1}{\sum_{v=1}^{n}\left(\theta_{v}-\beta_{i k}\right)^{2}+\frac{1}{v^{2}}}\right) \\
& \xi_{k} \mid \beta_{k}, \sigma^{2} \sim \mathrm{N}\left(\frac{\sum_{i=1}^{I_{k}} \beta_{i k}}{I_{k}}, \frac{\sigma^{2}}{I_{k}}\right) \\
& \omega_{k} \mid \alpha_{k}, v^{2} \sim \mathrm{N}\left(\frac{\sum_{i=1}^{I_{k}} \alpha_{i k}}{I_{k}}, \frac{v^{2}}{I_{k}}\right)
\end{aligned}
$$




$$
\begin{gathered}
\sigma^{2} \mid \beta_{k}, \xi_{k} \sim \operatorname{Inv}-\chi^{2}\left(\frac{\sum_{k=1}^{K} \sum_{i=1}^{I_{k}}\left(\beta_{i k}-\xi_{k}\right)^{2}+1}{\sum_{k=1}^{K} I_{k}+1}, \sum_{k=1}^{K} I_{k}+1\right) \\
v^{2} \mid \alpha_{k}, \omega_{k} \sim \operatorname{Inv}-\chi^{2}\left(\frac{\sum_{k=1}^{K} \sum_{i=1}^{I_{k}}\left(\alpha_{i k}-\omega_{k}\right)^{2}+1}{\sum_{k=1}^{K} I_{k}+1}, \sum_{k=1}^{K} I_{k}+1\right)
\end{gathered}
$$

\section{References}

Albert, J. H. (1992). Bayesian estimation of normal ogive item response curves using Gibbs sampling. Journal of Educational Statistics, 17, 251-269.

Angoff, W. H. (1971). Scales, norms, and equivalent scores. In R. L. Thorndike (Ed.), Educational measurement (2nd ed., pp. 508-600). Washington, DC: American Council on Education.

Bergan, J. R., \& Stone, C. A. (1985). Latent class models for knowledge domains. Psychological Bulletin, 98, 166-184.

Berk, R. A. (1980). A framework for methodological advances in criterion-referenced testing. Applied Psychological Measurement, 4, 563-573.

Berk, R. A. (1986). A consumer's guide to setting performance standards on criterionreferenced tests. Review of Educational Research, 56, 137-172.

Berk, R. A. (1996). Standard setting: The next generation (where few psychometricians have gone before!). Applied Measurement in Education, 9, 215-235.

Bradlow, E. T., Wainer, H., \& Wang, X. (1996). A Bayesian random effects model for testlets. Unpublished research report.

Chang, L., Dziuban, C. D., Hynes, M. C., \& Olson, A. H. (1996). Does a standard reflect minimal competency of examinees or judge competency? Applied Measurement in Education, 9, 161-173.

Cizek, G. J. (1993). Reconsidering standards and criteria. Journal of Educational Measurement, 30, 93-106.

Cizek, G. J. (1996a). Setting passing scores. Educational Measurement: Issues and Practice, 15(2), 20-31.

Cizek, G. J. (1996b). Standard-setting guidelines. Educational Measurement: Issues and Practice, 15(1), 13-21.

De Boeck, P., Daems, F., Meulders, M., \& Rymenams, R. (1997). Ontwikkeling van een toets voor de eindtermen begrijpend lezen [Construction of a test for the educational target of reading comprehension]. Leuven/Antwerp (Belgium): University of Leuven/ University of Antwerp.

Fischer, G. H. (1983). Logistic latent trait models with linear constraints. Psychometrika, 48, 3-26.

Gelman, A., Carlin, J. B., Stern, H. S. \& Rubin, D. B. (1995). Bayesian data analysis. New York: Chapman \& Hall. 
Gelman, A., \& Meng, X. L. (1996). Model checking and model improvement. In W. R. Gilks, S. Richardson, \& D. J. Spiegelhalter (Eds.), Practical Markov Chain Monte Carlo (pp. 189-201). New York: Chapman \& Hall.

Gelman, A., Meng, X. L., \& Stern, H. S. (1996). Posterior predictive assessment of model fitness via realized discrepancies (with discussion). Statistica Sinica, 6, 733-807.

Gelman, A., \& Rubin, D. B. (1992). Inference from iterative simulation using multiple sequences (with discussion). Statistical Science, 7, 457-511.

Gilks, W., Richardson, S. \& Spiegelhalter, D. (Eds.) (1996). Practical Markov Chain Monte Carlo. New York: Chapman \& Hall.

Glas, C.A.W. (1988). The derivation of some tests for the Rasch model from the multinomial distribution. Psychometrika, 53, 526-546.

Glass, G. V. (1978). Standards and criteria. Journal of Educational Measurement, 15, 237-261.

Hambleton, R. K. (1980). Test score validity and standard-setting methods. In R. A. Berk (Ed.), Criterion-referenced measurement: The state of the art (pp. 80-123). Baltimore: John Hopkins University Press.

Hambleton, R. K. (1982). Advances in criterion-referenced testing technology. In C. R. Reynolds \& T. B. Gutkin (Eds.), The handbook of school psychology (pp. 351-379). New York: Wiley.

Hambleton, R. K., \& Novick, M. R. (1973). Toward an integration of theory and method for criterion-referenced tests. Journal of Educational Measurement, 10, 159-170.

Huynh, H. (1976). Statistical consideration of mastery scores. Psychometrika, 41, 65-78.

Hoijtink, H., \& Molenaar, I. W. (1997). A multidimensional item response model: Constrained latent class analysis using the Gibbs sampler and posterior predictive checks. Psychometrika, 62, 171-189.

Jaeger, R. M. (1989). Certification of student competence. In R. L. Linn (Ed.), Educational measurement (3rd ed., pp. 485-514). New York: Macmillan.

Kane, M. (1994). Validating the performance standards associated with passing scores. Review of Educational Research, 64, 425-461.

Linn, R. L. (1980). Issues of validity for criterion-referenced measures. Applied Psychological Measurement, 4, 547-561.

Livingston, S. A., \& Zieky, M. J. (1982). Passing scores. Princeton, NJ: Educational Testing Service.

Lord, F. M., \& Novick, M. R. (1968). Statistical theories of mental test scores. Reading: Addison-Wesley.

Macready, G. B., \& Dayton, C. M. (1980). The nature and use of state mastery models. Applied Psychological Measurement, 4, 493-516.

Meng, X.-L. (1994). Posterior predictive p-values. Annals of Statistics, 22, 1142-1160.

Meskauskas, J. A. (1976). Evaluation models for criterion-referenced testing: Views regarding mastery in standard setting. Review of Educational Research, 45, 133-158.

Mislevy, R. J., Wingersky, M. S., \& Sheehan, K. M. (1994). Dealing with uncertainty about item parameters: Expected response functions (ETS Research Report 04204). Princeton, NJ: Educational Testing Service.

Nedelsky, L. (1954). Absolute grading standards for objective tests. Educational and Psychological Measurement, 14, 3-19.

Norcini, J. J. \& Shea, J. A. (1997). The credibility and comparability of standards. Applied Measurement in Education, 10, 39-59. 
Patz, R. J. (1996). Markov Chain Monte Carlo methods for item response theory models with applications for NAEP. Unpublished dissertation, Department of Statistics, Carnegie Mellon University, Pittsburgh, Pennsylvania.

Plake, B. S., Impara, J. C., \& Potenza, M. T. (1994). Content specificity of expert judgments in a standard-setting study. Journal of Educational Measurement, 31, 339-347.

Plake, B. S., \& Melican, G. J. (1989). Effects of item context on intrajudge consistency of expert judgments via the Nedelsky standard setting method. Educational and Psychological Measurement, 49, 45-51.

Rubin, D. B. (1984). Bayesianly justifiable and relevant frequency calculations for the applied statistician. Annals of Statistics, 12, 1151-1172.

Shepard, L. (1980). Standard setting issues and methods. Applied Psychological Measurement, 4, 447-467.

Shepard, L. A. (1994, October). Implications for standard setting of the NAE evaluation of NAEP achievement levels. Paper presented at the Joint Conference on Standard Setting for Large-scale Assessments. National Assessment Governing Board and $\mathrm{Na}-$ tional Center for Education Statistics, Washinghton, DC.

Spiegelhalter, D. J., Best, N. G., Gilks, W. R., \& Innip, H. (1996). Hepatitis B: A case study in MCMC methods. In W. R. Gilks, S. Richardson, \& D. J. Spiegelhalter (Eds.), Markov Chain Monte Carlo in practice (pp. 21-43). New York: Chapman \& Hall.

Tanner, M. A. (1996). Tools for statistical inference: Methods for the exploration of posterior distributions and likelihood functions (3rd ed.). New York: Springer.

van der Linden, W. J. (1982). A latent trait method for determining intrajudge inconsistency in the Angoff and Nedelsky techniques of standard setting. Journal of Educational Measurement, 19, 295-308.

Wilson, D., Wood, R., \& Gibbons, R. (1991). TESTFACT: Test scoring, item statistics, and item factor analysis. Chicago, IL: Scientific Software Inc.

Zellner, A., \& Min, C. (1995). Gibbs sampler convergence criteria. Journal of the American Statistical Association, 90, 921-927.

Zimowsky, M. R., Muraki, E., Mislevy, R. J., \& Bock, R. D. (1994). Bimain ${ }^{T M}$ 2: Multiple-group IRT analysis and test maintenance for binary items [Computer program]. Chicago, IL: Scientific Software International.

\section{Authors}

RIANNE JANSSEN is a postdoctoral researcher in the Department of Psychology at the University of Leuven, Tiensestraat 102, 3000 Leuven, Belgium; rianne.janssen@ped. kuleuven.ac.be. Her research interests include psychometric models.

FRANCIS TUERLINCKX is a Ph.D. student in the Department of Psychology at the University of Leuven, Tiensestraat 102, 3000 Leuven, Belgium; francis.tuerlinckx@ psy.kuleuven.ac.be. His research interests include psychometric models.

MICHEL MEULDERS is a Ph.D. student in the Department of Psychology at the University of Leuven, Tiensestraat 102,3000 Leuven, Belgium; michel.meulders@ psy.kuleuven.ac.be. His research interests include psychometric models.

PAUL DE BOECK is a professor in the Department of Psychology at the University of Leuven, Tiensestraat 102, 3000 Leuven, Belgium; paul.deboeck@psy.kuleuven.ac.be. His research interests include psychometric models. 
http://www.jstor.org

\title{
LINKED CITATIONS
}

- Page 1 of 3 -

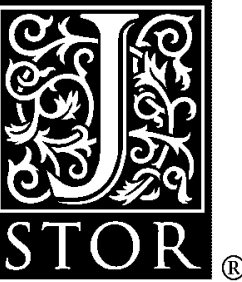

You have printed the following article:
A Hierarchical IRT Model for Criterion-Referenced Measurement
Rianne Janssen; Francis Tuerlinckx; Michel Meulders; Paul de Boeck
Journal of Educational and Behavioral Statistics, Vol. 25, No. 3. (Autumn, 2000), pp. 285-306.
Stable URL:
http://links.jstor.org/sici?sici=1076-9986\%28200023\%2925\%3A3\%3C285\%3AAHIMFC\%3E2.0.CO\%3B2-G

This article references the following linked citations. If you are trying to access articles from an off-campus location, you may be required to first logon via your library web site to access JSTOR. Please visit your library's website or contact a librarian to learn about options for remote access to JSTOR.

\section{References}

\author{
Bayesian Estimation of Normal Ogive Item Response Curves Using Gibbs Sampling \\ James H. Albert \\ Journal of Educational Statistics, Vol. 17, No. 3. (Autumn, 1992), pp. 251-269. \\ Stable URL: \\ http://links.jstor.org/sici?sici=0362-9791\%28199223\%2917\%3A3\%3C251\%3ABEONOI\%3E2.0.CO\%3B2-7
}

\author{
A Consumer's Guide to Setting Performance Standards on Criterion-Referenced Tests \\ Ronald A. Berk \\ Review of Educational Research, Vol. 56, No. 1. (Spring, 1986), pp. 137-172. \\ Stable URL: \\ http://links.jstor.org/sici?sici=0034-6543\%28198621\%2956\%3A1\%3C137\%3AACGTSP\%3E2.0.CO\%3B2-X
}

\author{
Reconsidering Standards and Criteria \\ Gregory J. Cizek \\ Journal of Educational Measurement, Vol. 30, No. 2. (Summer, 1993), pp. 93-106. \\ Stable URL: \\ http://links.jstor.org/sici?sici=0022-0655\%28199322\%2930\%3A2\%3C93\%3ARSAC\%3E2.0.CO\%3B2-V \\ Inference from Iterative Simulation Using Multiple Sequences \\ Andrew Gelman; Donald B. Rubin \\ Statistical Science, Vol. 7, No. 4. (Nov., 1992), pp. 457-472. \\ Stable URL: \\ http://links.jstor.org/sici?sici=0883-4237\%28199211\%297\%3A4\%3C457\%3AIFISUM\%3E2.0.CO\%3B2-Q
}


http://www.jstor.org

\section{LINKED CITATIONS}

- Page 2 of 3 -

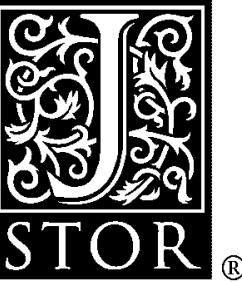

\section{Standards and Criteria}

Gene V. Glass

Journal of Educational Measurement, Vol. 15, No. 4, Special Issue on Standard Setting. (Winter, 1978), pp. 237-261.

Stable URL:

http://links.jstor.org/sici?sici=0022-0655\%28197824\%2915\%3A4\%3C237\%3ASAC\%3E2.0.CO\%3B2-\%23

\section{Toward an Integration of Theory and Method for Criterion-Referenced Tests}

Ronald K. Hambleton; Melvin R. Novick

Journal of Educational Measurement, Vol. 10, No. 3. (Autumn, 1973), pp. 159-170.

Stable URL:

http://links.jstor.org/sici?sici=0022-0655\%28197323\%2910\%3A3\%3C159\%3ATAIOTA\%3E2.0.CO\%3B2-Y

\section{Validating the Performance Standards Associated with Passing Scores}

Michael Kane

Review of Educational Research, Vol. 64, No. 3. (Autumn, 1994), pp. 425-461.

Stable URL:

http://links.jstor.org/sici?sici=0034-6543\%28199423\%2964\%3A3\%3C425\%3AVTPSAW\%3E2.0.CO\%3B2-O

\section{Posterior Predictive p-Values}

Xiao-Li Meng

The Annals of Statistics, Vol. 22, No. 3. (Sep., 1994), pp. 1142-1160.

Stable URL:

http://links.jstor.org/sici?sici=0090-5364\%28199409\%2922\%3A3\%3C1142\%3APP\%3E2.0.CO\%3B2-3

\section{Evaluation Models for Criterion-Referenced Testing: Views regarding Mastery and Standard-Setting}

John A. Meskauskas

Review of Educational Research, Vol. 46, No. 1. (Winter, 1976), pp. 133-158.

Stable URL:

http://links.jstor.org/sici?sici=0034-6543\%28197624\%2946\%3A1\%3C133\%3AEMFCTV\%3E2.0.CO\%3B2-V

\section{Content Specificity of Expert Judgments in a Standard-Setting Study}

Barbara S. Plake; James C. Impara; Maria T. Potenza

Journal of Educational Measurement, Vol. 31, No. 4. (Winter, 1994), pp. 339-347.

Stable URL:

http://links.jstor.org/sici?sici=0022-0655\%28199424\%2931\%3A4\%3C339\%3ACSOEJ\%3E2.0.CO\%3B2-W 
http://www.jstor.org

\section{LINKED CITATIONS \\ - Page 3 of 3 -}

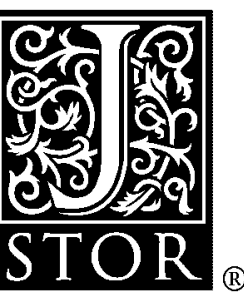

Bayesianly Justifiable and Relevant Frequency Calculations for the Applies Statistician Donald B. Rubin

The Annals of Statistics, Vol. 12, No. 4. (Dec., 1984), pp. 1151-1172.

Stable URL:

http://links.jstor.org/sici?sici=0090-5364\%28198412\%2912\%3A4\%3C1151\%3ABJARFC\%3E2.0.CO\%3B2-7

A Latent Trait Method for Determining Intrajudge Inconsistency in the Angoff and Nedelsky Techniques of Standard Setting

Wim J. Van Der Linden

Journal of Educational Measurement, Vol. 19, No. 4. (Winter, 1982), pp. 295-308.

Stable URL:

http://links.jstor.org/sici?sici=0022-0655\%28198224\%2919\%3A4\%3C295\%3AALTMFD\%3E2.0.CO\%3B2-0

\section{Gibbs Sampler Convergence Criteria}

Arnold Zellner; Chung-Ki Min

Journal of the American Statistical Association, Vol. 90, No. 431. (Sep., 1995), pp. 921-927.

Stable URL:

http://links.jstor.org/sici?sici=0162-1459\%28199509\%2990\%3A431\%3C921\%3AGSCC\%3E2.0.CO\%3B2-W 